\title{
Study on the Transducer Power Electronic Circuits Fault Diagnosis Based on SVM
}

\author{
Jianyong $\mathrm{Li}^{1, \mathrm{a}}$,Yanqiu Yang ${ }^{2, \mathrm{~b}}, \mathrm{Rui} \mathrm{Li}^{3, \mathrm{c}}, \mathrm{Yu} \mathrm{Lei}^{3, \mathrm{~d}}$
}

${ }^{1}$ Chongqing Lilong Technology Industry Group Co.Ltd, Chongqing China, 401123

${ }^{2}$ Chongqing Public Security Bureau, Chongqing, 401147

${ }^{3}$ Communication Sergeant School of the Army Engineering University, Chongqing, 400035

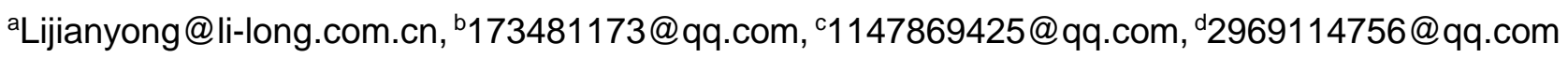

Keywords:SVM;Radial basis function; Transducer;Power electronic circuits;Fault diagnosis Abstract: The main circuit of inverter power transform fault mode analysis in the paper, using multiple support vector machine (SVM), implements the main circuit of inverter power transform fault diagnosis. In equipment fault diagnosis, online or offline to analyze the circuit, its running state, the learning ability of neural network, the fault parameters and the relationship between the cause of the problem through neural network learning after stored in its structure and power, you can through the analysis of fault parameters, it is concluded that the cause of the problem, so as to realize the automatic diagnosis of faults. Four characteristic parameters of reflecting fault state are distilled with analysis fault types and reasons of transducer power electronic circuits. Identification of fault mode can be completed utilizing multiply support vector machine(MSVM).

\section{Introduction}

Inverter obtained widespread application in the industrial production and daily life. Because of the complexity of the equipment, the operation personnel it is difficult to accurately grasp the equipment running situation, it often used after repair and preventive maintenance efficiency is not high maintenance. Reasonable maintenance should be unexpected in the presence of equipment fault early monitoring, so as to timely take measures reasonably. Predict the fault diagnosis technology for equipment maintenance.

\section{Fault types and causes}

Figure 1 is the inverter three-phase inverter main circuit; it is mainly composed of six power switch tube (MOSFET or IGBT) and the corresponding pulse driving circuit. Its common faults is switch tube damage or drive circuit malfunction. Fault types can be expressed as so:

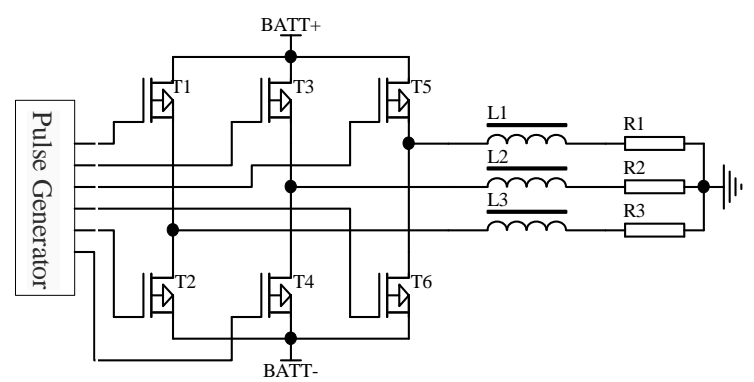

Fig1 three-phase inverter main circuit

(1) power switch tube base drive circuit fault; (2) power switch tube short circuit fault; (3) intermittent open circuit fault occurred in the power switch tube; (4) two power inverter bridge arm switch tube circuit fault; (5) two power inverter bridge arm switch tube fault; (6) passive devices (resistor, or inductance) fault in the circuit. 
Causes above all a common cause of fault are:(1) Each power switch tube usually need to separate the base driving circuit to drive in the high-power inverter, the fault is usually characterized by driving power supply fault. The fault causes switch tube control breakdown or open circuit. (2) Due to the large power switch tube under reverse breakdown caused by the reverse voltage caused, it will make the circuit of current surge, that cause overheating and damage to other components circuit. (3) The variation is due to the control circuit device performance or poor electromagnetic compatibility (such as self excitation circuit) because the power switch tube base drive fault. The fault can make the inverter output voltage waveform or cause overload and damage to other components. (4) Due to the base circuit malfunction. (5) The causes of such fault. Any power switch tube after a short circuit will cause another switch element stress also causes the short circuit. (6) Breakdown or gas leak explosion performance for capacitance and inductance saturation, the phenomenon such as resistor burn out, it will directly cause the inverter fail.

Actually we pay more attention to the switch tube of two short circuits and open circuit fault. The power switch tube of open circuit fault is used as an example for analysis in the paper. Consideration of actual system operation fault, in order to simplify the analysis process, we assume that at the same time there are two most bridge arm down, so the fault types can be divided into the following five categories: 22 small classes ${ }^{[1,2]}$.

Class 1: Normal operation of circuit, there is no power switch tube fault.

Class 2: there is a power switch tube fault occurs, it can be subdivided into six faults : (1)T1 fault, (2)T2 fault, (3)T3 fault,(4)T4 fault, (5)T5 fault, (6)T6 fault. Respectively 2, 3, 4, 5, 6, 7 classes.

Class 3: The half bridge with two power switch tube fault, it can be subdivided into three faults: (1)T1 and T2 fault, (2)T3 and T4 fault, (3)T5 and T6 fault. Respectively 8, 9, 10 types classes.

Class 4: The cross bridge of the two power switch tube fault, it can be subdivided into six faults: (1)T1 and T4, (2)T1 and T6 fault, (3)T3 and T6 fault, (4)T2 and T3 fault, (5)T4 and T5 fault, (6)T2 and T5 fault. Respectively 11, 12, 13, 14, 15, 16 classes.

Class 5:The same up(or below) bridge of the two power switch tube fault, it can be subdivided into six faults: (1)The T1 and T3 faults, (2) T1 and T5 faults, (3)T3 and T5 faults, (4)T2 and T4 faults, (5) T2 and T6 faults, (6)T4 and T6 faults. Respectively 17, 18, 19, 20, 21, 22 classes.

\section{Fault classification based on SVM}

SVM is based on the statistical learning theory developed a new type of learning Machine ${ }^{[3,4]}$, a small sample of learning Machine has the incomparable superiority, but in the practical application system. It is the cause using SVM rather than the other which the traditional classification such as neural networks in this paper.

Data for two kinds of points given training data set

$\left(x_{1}, y_{1}\right), \mathrm{L},\left(x_{l}, y_{l}\right), x \in R^{n}, \quad y \in(+1,-1)$

$l$ is the total number of training samples; $n$ is the dimensions of the pattern space; $y$ is the category. Nonlinear mapping the input vector $x$ is mapped to a high-dimensional feature space $Z$ using SVM by some prior choice, the optimal separating hyperplane is structured in this space, make the training data are separately hyperplane correctly, and the hyperplane nearest vector and the distance between the hyperplane is the biggest. SVM avoided directly deal with the problem by has the features of kernel function $K\left(x_{i}, x_{j}\right)$ in the high dimensional space.

In the paper uses a multiple category problem can be converted to two category problemtwo classification. Suppose you have samples $\left(x_{1}, y_{1}\right), \mathrm{L},\left(x_{l}, y_{l}\right), x \in R^{n}, y \in(1, \mathrm{~L}, k)$ said a sample class 
label, this is a problem that the sample is divided into $k$ classes. Two two classification method of turning it into $k(k-1) / 2$ two types of problems. For the class $i$ and class the first $j$ training data, we solve the following two category problem:

$$
\begin{aligned}
& \underset{w^{i j,}, b^{j}, \xi^{i j}}{\operatorname{Min}}\left[\frac{1}{2} w^{i j} \bullet \Phi\left(x_{t}\right)+C \sum_{i=1}^{s} \xi_{t}^{i j}\right] \\
& \left\{\begin{array}{cll}
w^{i j} \bullet \Phi\left(x_{t}\right)+b^{i j} \geq 1-\xi_{t}^{i j} & \text { for } \quad y_{t}=i \\
w^{i j} \bullet \Phi\left(x_{t}\right)+b^{i j} \leq-1+\xi_{t}^{i j} & \text { for } \quad y_{t}=j & \xi_{t}^{i j} \geq 0
\end{array}\right.
\end{aligned}
$$

$w^{i j}$ is the normal vector of the classification hyperplane; $\Phi(x)$ is the nonlinear mapping to a prior choice; $b^{i j}$ is the location of hyperplane; $\xi_{t}^{i j}$ the slack variable; $s$ is individual sum of $i$ class and $j$ class; $C$ is said compromise between classification interval and error rate.

By (2) and (3) can get $k(k-1) / 2$ decision function. When determining the $x$ category adopts the voting strategy ${ }^{[4]} \cdot f_{i j}(x)=w^{i j} \bullet \Phi(x)+b^{i j}>0, x$ belongs to $i$ class, vote for $j$ class. So on, all a decision function $k(k-1) / 2$ discriminated finally which kind of with the most votes just think $x$ what kind. When the phase at the same time, $x$ is classified as class standard small that ${ }^{[5]}$.

\section{Fault characteristic parameter acquisition and training}

Through the establishment of inverter main circuit simulation model and all kinds of fault experiment, can extract the fault feature vector. Open circuit fault simulation someone or some power tube can get rid of the drive signal of the switch tube equivalent implementation. If you choose the output voltage, current signal as the characteristic signal, since there are all voltage, current signal, and if the six-way signals as the characteristic signal to calculate the characteristic vector and feature vector data quantity will be very large, so that the SVM calculation of training it is quite complicated. The simulation fault signal analysis showed that, as long as selecting a voltage signal is basically can distinguish between the various circuit malfunction. The $V_{a b}$ signal is selected to obtain the fault characteristic information, by Fourier transform waveform signal transformation from time domain $V_{a b}$ to frequency domain, the use of the library functions of MATLAB analysis of $V_{a b}$ waveform signals, every harmonic amplitude and phase Angle can be obtained. Through analysis of every harmonic $V_{a b}$ waveform signal, the second harmonic (including DC component) of $V_{a b}$ signal can basically includes fault identification of all kinds of information. Therefore, choose DC component $(D)$, the amplitude values $A_{1}$, harmonics of phase Angle $\left(\Phi_{1}\right)$, two harmonic phase Angle $\left(\Phi_{2}\right)$ is as the characteristic signal, as the training sample of SVM. In order to reducing the amount of the data calculation, the characteristics of the signal data was normalized, which the sample data using the value to say.

Known from the analysis of the front, power electronics inverter main circuit fault types can be divided into five categories, a total of 22 small classes. The fault category number as shown in tab1:

Tab1 The fault code table

\begin{tabular}{|c|c|c|c|c|c|c|c|c|c|c|}
\hline normal & $\begin{array}{c}\mathrm{T} 1 \\
\text { fault }\end{array}$ & $\begin{array}{c}\mathrm{T} 2 \\
\text { fault }\end{array}$ & $\begin{array}{c}\mathrm{T} 3 \\
\text { fault }\end{array}$ & $\begin{array}{c}\mathrm{T} 4 \\
\text { fault }\end{array}$ & $\begin{array}{c}\mathrm{T} 5 \\
\text { fault }\end{array}$ & $\begin{array}{c}\mathrm{T} 6 \\
\text { fault }\end{array}$ & $\begin{array}{c}\mathrm{T} 1, \mathrm{~T} 2 \\
\text { fault }\end{array}$ & $\begin{array}{c}\mathrm{T} 3, \mathrm{~T} 4 \\
\text { fault }\end{array}$ & $\begin{array}{c}\mathrm{T} 5, \mathrm{~T} 6 \\
\text { fault }\end{array}$ & $\begin{array}{c}\mathrm{T} 1, \mathrm{~T} 4 \\
\text { fault }\end{array}$ \\
\hline Class 1 & Class 2 & Class 3 & Class 4 & Class 5 & Class 6 & Class 7 & Class 8 & Class 9 & Class 10 & Class 11 \\
\hline $\begin{array}{c}\mathrm{T} 1, \mathrm{~T} 6 \\
\text { fault }\end{array}$ & $\begin{array}{c}\mathrm{T} 3, \mathrm{~T} 6 \\
\text { fault }\end{array}$ & $\begin{array}{c}\mathrm{T} 2, \mathrm{~T} 3 \\
\text { fault }\end{array}$ & $\begin{array}{c}\mathrm{T} 4, \mathrm{~T} 5 \\
\text { fault }\end{array}$ & $\begin{array}{c}\mathrm{T} 2, \mathrm{~T} 5 \\
\text { fault }\end{array}$ & $\begin{array}{c}\mathrm{T} 1, \mathrm{~T} 3 \\
\text { fault }\end{array}$ & $\begin{array}{c}\mathrm{T} 1, \mathrm{~T} 5 \\
\text { fault }\end{array}$ & $\begin{array}{c}\mathrm{T} 3, \mathrm{~T} 5 \\
\text { fault }\end{array}$ & $\begin{array}{c}\mathrm{T} 2, \mathrm{~T} 4 \\
\text { fault }\end{array}$ & $\begin{array}{c}\mathrm{T} 2, \mathrm{~T} 6 \\
\text { fault }\end{array}$ & $\begin{array}{c}\mathrm{T} 4, \mathrm{~T} 6 \\
\text { fault }\end{array}$ \\
\hline Class 12 & Class 13 & Class 14 & Class 15 & Class 16 & Class 17 & Class 18 & Class 19 & Class 20 & Class 21 & Class 22 \\
\hline
\end{tabular}

So, the circuit fault classification can be classified into 231 two types of classification problem, then identified into 22 class fault identification using voting strategy. Among the kernel function is nonlinear transform using radial basis kernel function:

$$
K\left(x, x_{i}\right)=\exp \left(\frac{\left(x-x_{i}\right)^{T}\left(x-x_{i}\right)}{2 \sigma^{2}}\right)
$$


Setting $C=10000, \sigma=10$. Using MATLAB simulation inverter main circuit fault, 22 classes were taken under the fault of 176 groups, among 110 groups used for training SVM and other 66 groups for simulation.

\section{Simulation verification}

The simulation data as a feature vector, the input above the established many kind of SVM, which represents 22 such fault of 22 groups of data validation output as shown in table 2:

Tab2 Simulation data output table

\begin{tabular}{|c|c|c|c|c|c|c|c|c|c|c|c|c|c|c|c|c|c|c|c|c|c|}
\hline 1 & 2 & 3 & 4 & 5 & 6 & 7 & 8 & 9 & 10 & 11 & 12 & 13 & 14 & 15 & 16 & 17 & 18 & 19 & 20 & 21 & 22 \\
\hline 21 & 11 & 5 & 8 & 11 & 16 & 10 & 15 & 8 & 19 & 7 & 16 & 15 & 3 & 14 & 4 & 14 & 3 & 8 & 14 & 0 & 9 \\
\hline 17 & 21 & 9 & 14 & 16 & 12 & 10 & 13 & 7 & 15 & 17 & 20 & 10 & 4 & 8 & 4 & 11 & 4 & 5 & 7 & 0 & 7 \\
\hline 9 & 9 & 21 & 13 & 8 & 6 & 11 & 5 & 13 & 13 & 9 & 14 & 16 & 17 & 4 & 19 & 3 & 2 & 18 & 1 & 20 & 0 \\
\hline 12 & 13 & 14 & 21 & 10 & 8 & 14 & 10 & 15 & 14 & 13 & 16 & 20 & 13 & 6 & 7 & 4 & 3 & 15 & 2 & 1 & 0 \\
\hline 14 & 14 & 7 & 10 & 21 & 12 & 6 & 8 & 4 & 9 & 15 & 11 & 8 & 2 & 20 & 3 & 13 & 17 & 2 & 16 & 0 & 19 \\
\hline 16 & 11 & 4 & 8 & 14 & 21 & 9 & 13 & 4 & 10 & 11 & 10 & 8 & 2 & 17 & 3 & 14 & 16 & 4 & 20 & 0 & 16 \\
\hline 18 & 12 & 8 & 12 & 10 & 14 & 21 & 12 & 9 & 16 & 8 & 16 & 18 & 11 & 6 & 7 & 3 & 3 & 12 & 12 & 1 & 2 \\
\hline 16 & 12 & 6 & 8 & 12 & 13 & 6 & 21 & 5 & 11 & 6 & 14 & 12 & 2 & 19 & 3 & 18 & 15 & 3 & 15 & 0 & 14 \\
\hline 18 & 9 & 5 & 8 & 15 & 15 & 5 & 10 & 8 & 12 & 10 & 10 & 12 & 2 & 19 & 3 & 18 & 12 & 7 & 16 & 0 & 17 \\
\hline 4 & 10 & 10 & 15 & 9 & 13 & 15 & 11 & 15 & 20 & 8 & 16 & 19 & 11 & 6 & 7 & 3 & 3 & 13 & 10 & 2 & 1 \\
\hline 14 & 18 & 7 & 13 & 17 & 12 & 7 & 9 & 5 & 10 & 21 & 14 & 9 & 4 & 7 & 3 & 12 & 19 & 3 & 6 & 1 & 20 \\
\hline 18 & 20 & 9 & 13 & 15 & 12 & 8 & 13 & 8 & 16 & 13 & 21 & 11 & 4 & 8 & 4 & 11 & 5 & 5 & 7 & 0 & 10 \\
\hline 12 & 13 & 13 & 20 & 10 & 8 & 14 & 10 & 14 & 13 & 13 & 17 & 21 & 14 & 6 & 7 & 4 & 3 & 16 & 2 & 1 & 0 \\
\hline 8 & 13 & 18 & 15 & 7 & 6 & 10 & 5 & 13 & 11 & 10 & 14 & 13 & 21 & 4 & 17 & 3 & 3 & 20 & 1 & 19 & 0 \\
\hline 13 & 13 & 6 & 10 & 20 & 14 & 5 & 10 & 4 & 11 & 12 & 10 & 8 & 2 & 21 & 3 & 15 & 17 & 2 & 17 & 0 & 18 \\
\hline 10 & 9 & 19 & 13 & 7 & 7 & 11 & 5 & 13 & 13 & 8 & 14 & 17 & 17 & 4 & 21 & 3 & 2 & 18 & 1 & 19 & 0 \\
\hline 16 & 13 & 8 & 12 & 11 & 8 & 9 & 10 & 10 & 18 & 8 & 17 & 17 & 11 & 16 & 5 & 19 & 3 & 10 & 2 & 0 & 8 \\
\hline 13 & 18 & 7 & 12 & 15 & 9 & 7 & 11 & 5 & 12 & 20 & 17 & 9 & 4 & 7 & 3 & 12 & 21 & 3 & 6 & 1 & 19 \\
\hline 9 & 9 & 18 & 15 & 8 & 6 & 11 & 5 & 13 & 11 & 10 & 14 & 15 & 19 & 4 & 17 & 3 & 2 & 21 & 1 & 20 & 0 \\
\hline 14 & 12 & 6 & 8 & 16 & 13 & 5 & 14 & 4 & 10 & 11 & 11 & 9 & 2 & 19 & 3 & 18 & 15 & 3 & 21 & 0 & 17 \\
\hline 9 & 10 & 20 & 15 & 8 & 6 & 10 & 6 & 12 & 11 & 10 & 14 & 15 & 19 & 4 & 18 & 3 & 2 & 17 & 1 & 21 & 0 \\
\hline 15 & 19 & 8 & 14 & 16 & 11 & 8 & 9 & 7 & 13 & 20 & 17 & 11 & 5 & 8 & 4 & 13 & 4 & 3 & 5 & 1 & 20 \\
\hline
\end{tabular}

The output matrix to pay online count in their respective columns is basically in maximum (except 9 columns) from table 2. So using multiple class support vector machines for power electronics inverter main circuit fault recognition rate is as high as $95 \%$, and has nothing to do with the training sample number, fully embodies the under the condition of small samples, the advantages of SVM in fault pattern recognition.

\section{Conclusion}

When using radial basis kernel function, implementation of SVM is a kind of radial basis function (RBF) classifier. However, the center location, number and network weights of RBF are automatically determined by the training process, no longer relies on the experience of expert knowledge. It is a new way to solve the problem of the fault diagnosis of complicated systems. More class support vector machines are used to achieve the fault mode of pattern recognition.

\section{References}

[1] Dehong $\mathrm{Xu}$, Hao Ma. Power electronic equipment fault diagnosis automatically[M]. Beijing: Science press, 2010

[2] Minyuan Li. A controlled rectifier circuit fault diagnosis method based on pattern recognition, Journal of electrotechnics,2009,19(7):53-58

[3] Kaili Zhou, Chunhui Zhou. Tutorial MATLAB and its application[M]. Beijing: Beijing university press, 2007 
[4] Hsu Ch.W, Lin ChJ. A comparison of methods for multi-2 class support vector machines[J]. IEEE Transactions on Neural Networks,2012,13(3) :415 425.

[5] K.Mohammadi, S.J.Seyyed Mahdavi. On improving training time of neural networks in mixed signal circuit fault diagnosis applications[J]. Microelectronocs Reliability,2008 